\title{
The effect of stacking fault energy on acoustic emission in pure metals with face-centered crystal lattice
}

\author{
A. V. Danyuk ${ }^{1, \dagger}$, D. L. Merson ${ }^{1}$, I.S. Yasnikov ${ }^{1}$, E. A. Agletdinov ${ }^{1}$, M. A. Afanasiev${ }^{1}$, \\ A. Vinogradov ${ }^{1,2}$ \\ †alexey.danyuk@gmail.com
}

\author{
${ }^{1}$ Togliatti State University, ul. Belorusskaya, 14, Togliatti, 445020, Russia \\ ${ }^{2}$ Norwegian University of Science and Technology, 7491 Trondheim, Norway
}

\begin{abstract}
Many questions remain open in the understanding the role of microstructural factors in the acoustic emission (AE) phenomenon occurring in deforming materials. A comparative analysis of AE time parameters in tensile testing of pure aluminum, copper, silver and nickel specimens having very different values of stacking fault energy (SFE) was undertaken in the present work to clarify the SFE effect on the AE signal. Continuous digital wideband recording was used for AE waveform registration, which offers the possibility to avoid the threshold discriminators and to analyze a continuous AE signal generated during plastic deformation mediated by dislocation mechanisms. The power of the AE signal were selected as the descriptive parameters. Following the evolution of dislocation structures, the AE energy parameters were demonstrated to have a similar behavior in all investigated materials, i.e. the AE level increases sharply at the onset of plastic flow and then decays gradually during the uniform strain hardening stage. However, the absolute values of the AE amplitude and energy differ significantly depending on SFE. It was shown unambiguously that in contrast to expectations, the AE energy parameters reduce as the SFE value increases. This effect is discussed qualitatively in terms of the features of dislocation behavior, which are governed by the SFE value.
\end{abstract}

Keywords: acoustic emission, stacking fault energy, deformation of pure metals, FCC metals, dislocation slip.

\section{Introduction}

Currently the multiple of methods and research instruments enable the study of the deformation behavior of crystalline materials at all stages from the early elastic strains to failure, and at all structural levels from the movement of single dislocations to critical instability and complete fracture.

This study aims at exploring the effect of the stacking fault energy (SFE) on the acoustic emission (AE) signal recorded during monotonic tensile tests of pure metal specimens under identical experimental conditions.

Attempts to describe the $\mathrm{AE}$ in different pure polycrystalline metals are found in many studies, for example, in [1-5] for FCC, [6-8] for BCC and [9-11] for HCP metals. However, (i) the authors of early papers have focused usually on one or two materials that did not allow for any comparative analysis and generalization for the entire class of materials with the same crystallographic lattice, and (ii) the equipment used and the methods of signal registration and analysis prevented from performing quantitative comparative assessments. Furthermore, a comparative analysis of even a simple amplitude level of AE signal obtained by different authors for different metals cannot be completed due to substantial differences in sensor sensitivity, non-identical equipment settings, different experiment conditions (e.g. the strain rate), geometry and metallographic characteristics of the specimens tested. The modern systems for AE data recording and analysis have incomparably better capabilities than the equipment used in 1970s-1990s. This provides much more ample analytical options for the $\mathrm{AE}$ method when applied to materials science [12]. This accounts for a significant rise of interest that is recently seen in the literature regarding the $\mathrm{AE}$ method as a unique tool for investigating the deformation micromechanics in modern structural materials and as well as for fracture prediction based on fundamental understanding of the features of microstructure evolution and transition to a critical state just before failure.

The evolution of dislocation microstructure and the related staging of deformation in pure polycrystalline metals with FCC lattice has been well understood [13]. The investigation and comparison of deformation behaviors of different crystalline materials with the concomitant recording and analyzing the AE signal is of great interest nowadays, because the development of adequate microstructure-based models of AE phenomenon would substantially enhance the predictive capability of the AE technology used in modern materials science laboratory systems and/or in operational diagnostic systems for in-field nondestructive integrity testing. 
One of least explored and understood problems is the effect of SFE on the AE signal. The SFE is the material property that determines the nature of the dislocation slip (planar or wavy) due to different capabilities of dislocations for cross-slip and climb (at high temperatures under creep conditions), i.e. due to their different mobilities [14]. In Hatano's early paper [15], the SFE effect was investigated using copper, alpha-brass (70Cu-30Zn), stainless steel polycrystals (304: $18 \mathrm{Cr}-8 \mathrm{Ni})$, as well as single crystals of $\mathrm{Cu}$ and $\mathrm{Cu}-\mathrm{Al}$ alloy $(\mathrm{Cu}-\mathrm{Al}$ and $\mathrm{Cu}-0.34-1.15 \mathrm{Al}$ - all in weight \%). A conclusion was made about the significant drop in the AE signal amplitude with decreasing SFE of the material. Hsu et al. [16] proposed to relate the AE energy gain to the SFE increase through eased cross-slip in materials with higher SFE. In such materials, the tendency to form cellular structures with significant free space for dislocation slip is clearly visible. It is due to a larger free path of dislocations that AE signal increase is expected. Such an interpretation was challenged by Heiple and Carpenter [17], who noted the fact that the formation of a cellular structure occurs at the mature deformation, while the AE peak is observed at much lower strains, less (or even much less) than $0.2 \%$. However, it is noteworthy that the choice of the materials investigated by Hatano can hardly be considered proper to draw reasonable conclusions on the role of SFE on AE because of a complex effect which alloying exerts on the deformation behavior and on the concomitant $\mathrm{AE}[5,18]$. In fact, solid solution strengthening which is very significant in the $\mathrm{Cu}$-based alloys or austenitic stainless steel used in [15] affects the AE behavior in the same way as the SFE does, i.e. AE decreases significantly with increasing alloying.

Hence, the objective of the present report is to investigate and clarify the effect of SFE on the AE signal during plastic deformation of pure metals.

The paper describes the deformation behavior of FCC metals exemplified by aluminum, copper and silver of nominal purity better than $99.99 \%$, and nickel of technical purity. The choice of those elements is explained by the fact that they have significantly different SFE values ranging from about $300 \mathrm{~mJ} / \mathrm{m}^{2}$ for $\mathrm{Ni}$ to $20 \mathrm{~mJ} / \mathrm{m}^{2}$ for Ag (Table 1). These materials are therefore the most common in the studies that attempted to assess the influence of SFE on the deformation hardening of metals and alloys [19].

Table 1. Properties of metals and experimental results.

\begin{tabular}{|c|c|c|c|c|}
\hline \multirow{2}{*}{ Properties } & \multicolumn{4}{|c|}{ Metal } \\
\cline { 2 - 5 } & $\mathrm{Ni}$ & $\mathrm{Al}$ & $\mathrm{Cu}$ & $\mathrm{Ag}$ \\
\hline $\begin{array}{c}\text { Stacking Fault } \\
\text { Energy, } S F E \gamma, \mathrm{mJ} / \mathrm{m}^{2} \\
{[14,23][24][25]}\end{array}$ & 300 & $170-250$ & $40-70$ & $18-25$ \\
\hline $\begin{array}{c}\text { Mean grain size, } \\
d, \mu \mathrm{m}\end{array}$ & $500 \pm 300$ & $200 \pm 100$ & $100 \pm 35$ & $90 \pm 70$ \\
\hline $\begin{array}{c}\text { Elongation to } \\
\text { failure, } \varepsilon\end{array}$ & 0,40 & 0,50 & 0,47 & 0,49 \\
\hline $\begin{array}{c}\text { Amplitude of } \mathrm{AE} \\
\text { peak, } A_{A E m a x} \mu \mathrm{V}\end{array}$ & 31 & 36 & 46 & 57 \\
\hline $\begin{array}{c}\text { Peak of } \mathrm{AE} \text { power, } \\
P_{A E m a x} \mu \mathrm{V}^{2}\end{array}$ & 30 & 43 & 82 & 126 \\
\hline
\end{tabular}

\section{Experiment Materials and Methods}

Identical flat tensile specimens having the gauge length of $15 \times 7 \times 2 \mathrm{~mm}$ and wide shoulders designed for mounting the AE sensor and gripping in the testing machine were cut from $2 \mathrm{~mm}$ thick pure metal plates $(\mathrm{Ni}, \mathrm{Al}, \mathrm{Cu}, \mathrm{Ag}$ ) (produced by Nilaco Corporation, Japan) using electric spark cutting. Before testing, the specimens were annealed in vacuum for 90 minutes at a temperature of $0.85 \cdot T_{m}$, where $T_{m}$ is melting point of the relevant metal. As a result, the coarse grain specimens were produced for all elements with comparable grain size from 90 to $500 \mu \mathrm{m}$, Fig. 1. The grain structure was assessed on electrolytically polished specimens by using optical and scanning electron microscopy (SEM) and using the electron back scattered diffraction technique (EBSD). The EBSD detector Hikari-5 EDAX/TSL was installed in the SEM Zeiss SIGMA equipped with a field emission gun.

Tensile samples were tested on a universal testing machine Tinius Olsen H50KT with a $5 \mathrm{kN}$ load cell and a precision strain measurement system using an extensometer with $1 \mu \mathrm{m}$ resolution. Samples were tested at constant crosshead speed $2 \mathrm{~mm} / \mathrm{min}$, which corresponded to a nominal strain rate of $2 \cdot 10^{-3} \mathrm{~s}^{-1}$. It should be noted that the testing machine used has a low level of acoustic noise, and special grips were designed to fix miniature specimens to avoid any possible noise from friction or similar effects caused by the loading frame.

The acoustic emission signal was recorded by a wideband piezoelectric transducer NF AE-900S WB in the frequency band from 100 to $900 \mathrm{kHz}$. The transducer was connected to a low noise amplifier PAC 2/4/6 with gain $+60 \mathrm{~dB}$ and the AE signal was recorded by a PAC (Physical Acoustic Corporation, USA) PCI-2 board that was configured for continuous waveform recording (non-threshold recording mode) with additional gain of $+6 \mathrm{~dB}$ and band-pass filtering $10 \div 1000 \mathrm{kHz}$, the sampling frequency of the digital recording was $2 \mathrm{MHz}$.
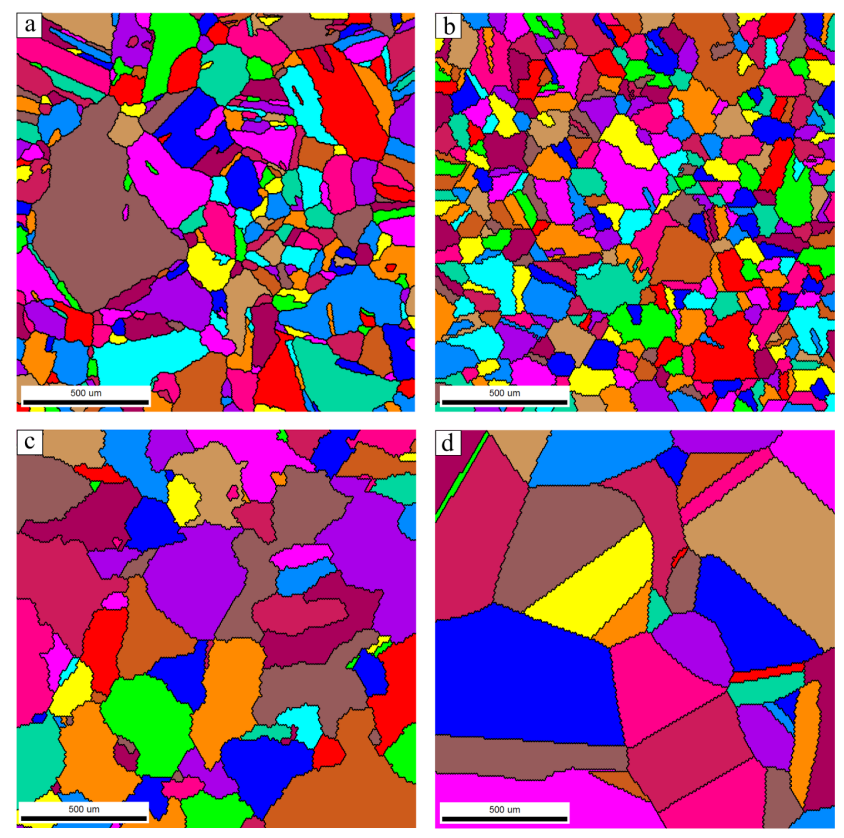

Fig. 1. (Color online) The grain structure of various samples of pure metals - EBSD grain map: (a) - Ag; (b) - Cu; (c) - Al; (d) - Ni. 


\section{Results and interpretation}

Initial results of tensile tests on specimens of the materials studied are presented in Fig. 2. The shape of mechanical tensile diagrams for all materials is typical for pure metals with the FCC lattice and different SFE values: there is a significant difference between the yield stresses corresponding to the onset of plastic flow in different materials, their tensile ultimate strength, the lack of yield plateau, and relatively high ductility. The amplitude of the continuously recorded AE signal also exhibits the shape characteristic of pure annealed FCC materials: the presence of distinctive sharp maximum (peak) of amplitude in the beginning of plastic flow, and its subsequent gradual monotonic decay as the strain increases. It is worth noting that all selected pure FCC materials without hardening elements have the SFE exceeding $15-20 \mathrm{~mJ} / \mathrm{m}^{2}$ [20], which is high enough to inhibit mechanical twinning at room temperature.

Despite the discrete nature of dislocation slip lines, the recorded $\mathrm{AE}$ signal arising in response to profuse slip is continuous as many dislocation movements occur per recording unit time. Thus, distinguishing discrete AE events that correspond to generation of individual slip events makes no sense. The implemented in the present work continuous thresholdless $\mathrm{AE}$ acquisition enables evaluation and comparison of AE parameters at any time of the experiment. The use of a time interval of any desired length is beneficial for further processing and for obtaining the statistically relevant results. The average power of $\mathrm{AE}$ signal $P_{A E}$ (referred to $1 \mathrm{Ohm}$ nominal impedance) was calculated from the initial continuous "streams" sectioned by the sliding time window $\Delta t$ of 500 ms duration, as:
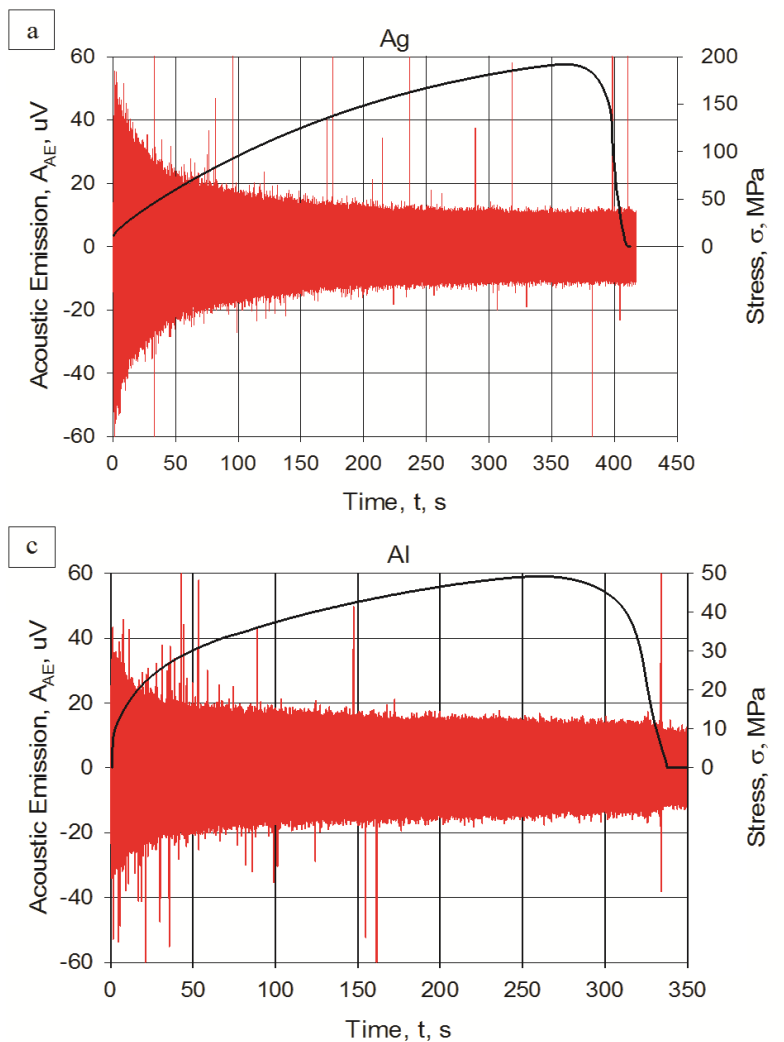

$$
P_{A E}(t)=\frac{1}{N} \sum_{i=0(t)}^{N(t+\Delta t)}\left(u_{A E}(i)\right)^{2}
$$

where $t$ denotes the current time from the beginning of test; $u_{A E}(i)$ is the $i$-th reading of the amplitude of the AE signal in the window; $N(\Delta t)$ is the size of the sliding window (number of samples in the time interval $\Delta t$ ) Being an adequate characteristic of the random $\mathrm{AE}$ time series, the $\mathrm{AE}$ power in this formulation virtually excludes the impact of transient noise signals (spontaneous spikes etc.) or rare fluctuations of acoustic events. Fig. 3 demonstrates the synchronized data of the AE power, $P_{A E}$, with loading curve in the true strain-stress coordinates.

The AE power $P_{A E}$ for all samples, Fig. 3. shows a similar behavior resembling qualitatively (but surely not quantitatively) the trends in the signal amplitude behavior seen in Fig. 1. At the stage of maximum strain hardening rate, $P_{A E}$ reaches its maximum, and then monotonically decreases (but does not vanish completely) until the final fracture. A slight increase in the $P_{A E}$ value is observed shortly before fracture that is associated with the significant increase of the local strain rate in the specimen neck and the formation of fracture micro-cracks. An important feature of the $\mathrm{AE}$ behavior, which is worthy noting, is the correlation between the strain hardening coefficient, $\theta=\mathrm{d} \sigma / \mathrm{d} \varepsilon$, and $\mathrm{AE}$ power for all selected alloys (Fig. 3). It is manifested in the fact that the peak of the AE power, $P_{\text {AEmax }}$ is observed in the earliest deformation stages where the most rapid hardening occurs, and then the $\mathrm{AE}$ power decreases along with the $\theta$ reduction. Also note that nickel demonstrates the presence of the second stage of deformation - linear hardening stage, where the value of $\theta$ is constant, whereas in other studied metals this

Fig. 2. (Color online) AE amplitude (red) and strain - stress curve (black): (a) - Ag; (b) - Cu; (c) - Al; (d) - Ni.

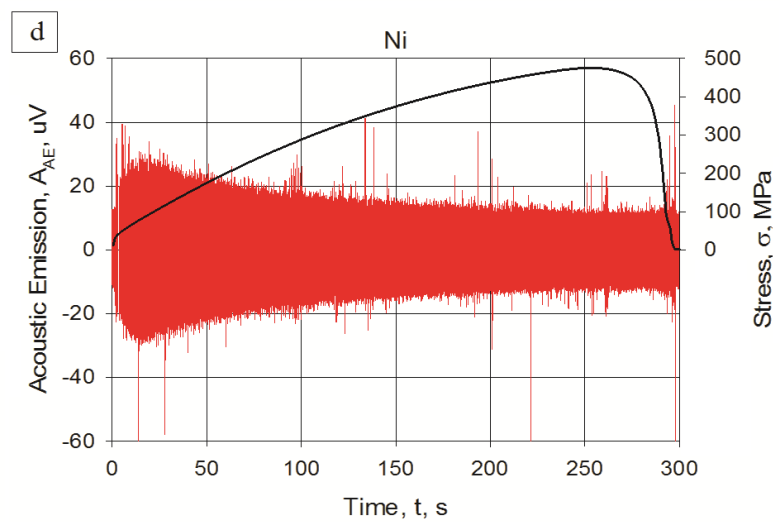



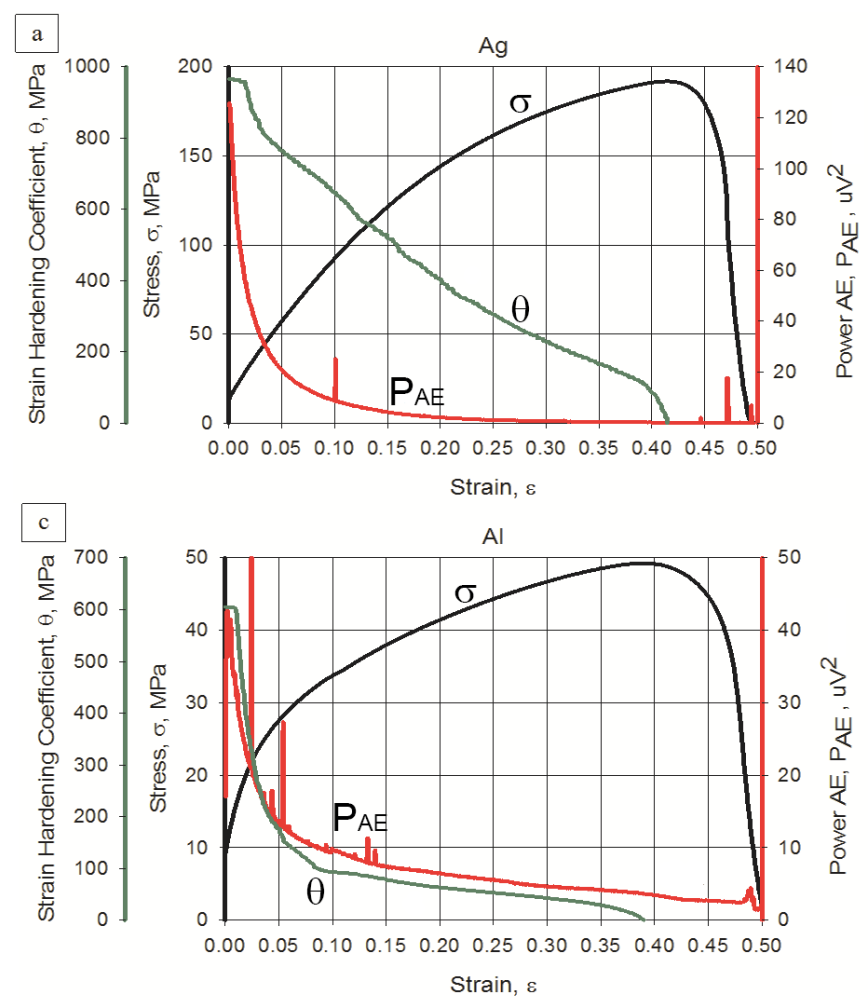
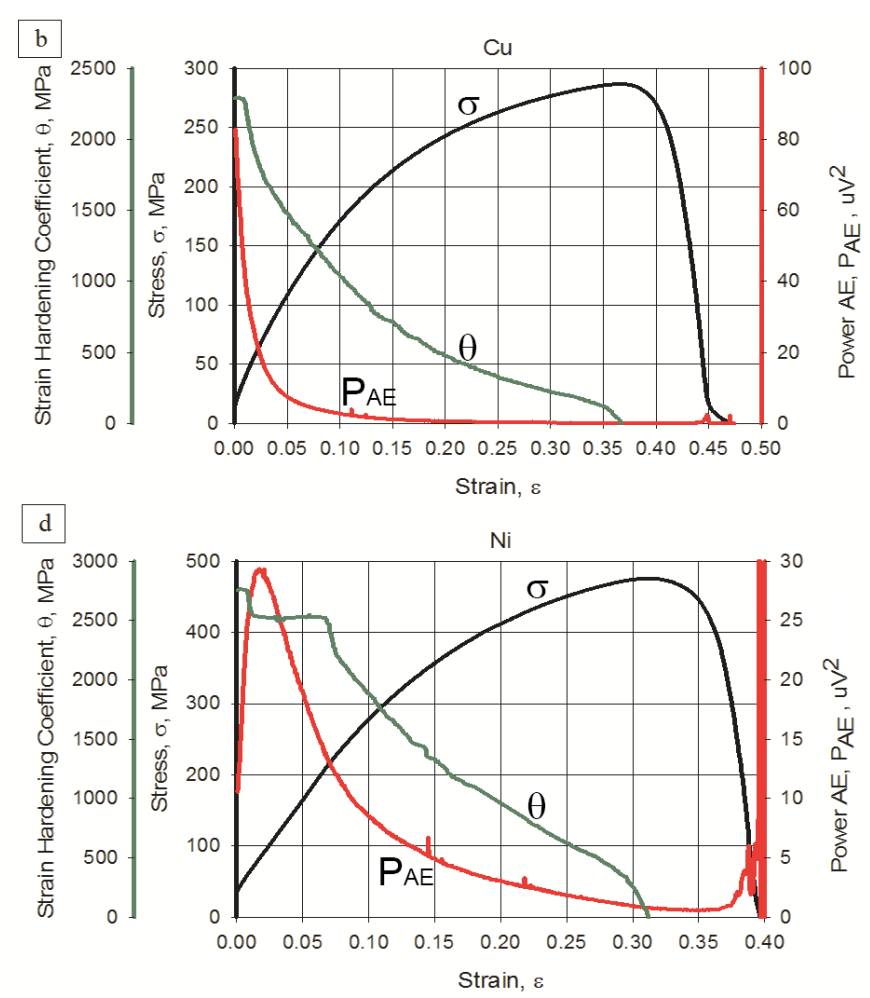

Fig. 3. (Color online) Hardening curve (black), strain hardening coefficient (green), and AE power (red) for different samples: (a) — Ag; (b) $-\mathrm{Cu} ;(\mathrm{c})-\mathrm{Al} ;(\mathrm{d})-\mathrm{Ni}$.

stage is degenerated. Most likely, this is due to the presence of a few very large grains in nickel (see Fig. 1d). These grains, while spreading almost across the entire thickness of the specimen, behave like single crystals, in which this stage is most clearly visible. The correlation between $P_{A E}$ and $\theta$ for copper was noted in several earlier papers [21] [22], though in apparently a less conclusive way. Generalization of this empirical feature, which has been systematically observed in the present work, is a crucial finding for understanding of the nature and phenomenological modeling of the $\mathrm{AE}$ phenomenon that will be presented elsewhere.

Relevant characteristics are summarized in Table 1, which permits analyzing the relationship between $\mathrm{AE}$ and deformation characteristics of a given metal. SFE is a physical parameter that largely determines the kinetics of dislocations, i.e. their generation, motion, annihilation and storage rate during plastic deformation, and materials mechanical properties. The total elongation (strain) to fracture is an indicator of the material's ductility, and the AE amplitude peak and the height of AE power peak are the parameters of source activity.

Analysis of the dependence of the AE power peak on SFE (Fig. 4) reveals that the maximum value, $P_{\text {AEmax }}$, undoubtedly decreases as SFE increases, with other microstructural and texture characteristics of the materials being similar. So, this dependence is opposite to that described in the cited Hatano's early study. Therefore, since only pure well-annealed metals were used in the present study, the effect that was observed in the aforementioned paper appears to be attributable to the impact of impurities and to the related solid-solution hardening rather than to the role of SFE. The value of $P_{\text {AEmax }}$ found for nickel may raise some doubts, Fig. 4, because unlike other tested metals nickel used in the present work is of technical grade and is not the very high purity material, while the impurities are known $[5,18]$ to reduce the height of AE peak. On the other hand, the nickel grain size in our case is bigger than in other metals which with sufficiently large grain size should on the contrary lead to the AE energy gain, as was observed, for example, in pure $\mathrm{Al}$ [1]. Hence, the $\mathrm{AE}$ energy loss with increasing SFE trend, most likely, is true for nickel as well.

The SFE affects many materials properties. First of all, it determines the nature of strain hardening [25]. The high SFE implies a smaller size of the dislocation core and, conversely, a decreased SFE leads to an increase of the dislocation core width, which significantly changes the behavior of the metal in the whole range of strains. A higher value of SFE means that dislocation mobility increases. Because of the smallness of dislocation core size with a high SFE in each slip plane at the early stage of strain much greater number of dislocations can accumulate than with a low SFE, due to a smaller elastic stress field they can get closer to each other. As a consequence,

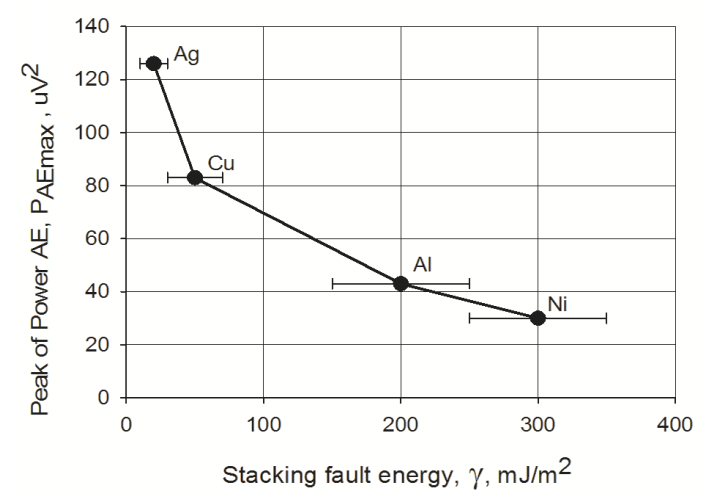

Fig. 4. Dependence of maximum AE power on the stacking fault energy in pure metals. 
the less the free path, the higher is the probability of opposite sign screw components collision, and, accordingly, the higher is the frequency of their annihilation and the rate of dynamic recovery [26]. The lower SFE (larger size of dislocation core), on the contrary, increases the distance between partial dislocations and makes them less mobile. Materials with low SFE exhibit wider stacking faults making difficult the crossslip and climb of dislocations and, accordingly, hindering the annihilation of screw dislocations of opposite sign in parallel planes. This leads to higher rates of accumulation of dislocations in the bulk that was observed by many researchers $[27,28]$, and thus contributes to the AE signal energy buildup. The qualitative explanation of the obtained results can be found on the basis of the paper by Copley and Kear [29]. These authors analyzed the dependence of splitting of Shockley partial dislocations in the FCC lattice on the instantaneous rate of dislocations. In accordance with their model predictions, the rate of partial dislocations increases significantly with the increase of partials' splitting, i.e. with the SFE decrease.

\section{Conclusion}

Qualitatively similar behavior of the acoustic emission parameters for different metals studied, $\mathrm{Ag}, \mathrm{Cu}, \mathrm{Al}$ and $\mathrm{Ni}$, is a result of an identical deformation mechanism in the FCC lattice, i.e. dislocation slip. In this case, the AE energy parameters reflect the evolution of dislocation ensembles, revealing corresponding stages. However, $\mathrm{AE}$ quantitative characteristics are very sensitive to the stacking fault energy, because the latter exerts a significant impact on the dislocation kinetics. The use of pure metals and common methodical approach to data acquisition and processing allows us to conclude that in contrast to existing early assumptions the higher SFE results in a decrease of the AE amplitude and energy (power) peak magnitude. The physical nature of the observed phenomenon has yet to be clarified and taken into account in attempts to model the AE phenomenon either phenomenologically or microscopically.

Acknowledgements. Financial support from the State Assignment of the Ministry of Science and Education of Russia according to the contract N 11.5281.2017/8.9 is gratefully appreciated.

\section{References}

1. C. Scruby, H. Wadley, J.E. Sinclair, Philosophical Magazine A 44 (2) (1981) 249-274.

2. J. Baram, M. Rosen, Materials Science and Engineering 47 (3) (1981) $243-246$.

3. Z. I. Bibik, Fizika Metallov I Metallovedenie 63 (4) (1987) $811-815$.

4. Z.I. Bibik, Fizika Metallov I Metallovedenie 59 (4) (1985)
$822-826$

5. M.A. Krishtal, D.L. Merson, A.V. Katsman, M. A. Vyboyschchik, Phys Met Metallogr+ 66 (3) (1988) $169-175$.

6. F. P. Higgens, S.H. Carpenter, Acta Metallurgica 26 (1) (1978) $133-139$.

7. V.A. Strizhalo, M.V. Kalashnik, S.I. Likhatskii, I. N. Ponomarenko, V. I. Belogurova, Strength of Materials 15 (11) (1983) $1528-1531$.

8. A. Lazarev, A. Vinogradov, J. of Acoustic Emission 27 (2009) $144-156$.

9. P. Dobroň, J. Bohlen, F. Chmelík, P. Lukáč, D. Letzig, K. U. Kainer, Materials Science and Engineering A 462 (1-2) (2007) $307-310$.

10. M. Friesel, S. Carpenter, Metall and Mat Trans A 15 (10) (1984) $1849-1853$.

11. J. Dosoudil, Z. Trojanova, P. Lukac, F. Chmelik, Plasticity of Metals and Alloys - ISPMA 6 (1994) 401 - 406.

12. A. Vinogradov, I. S. Yasnikov, Y. Estrin, Journal of Applied Physics 115 (23) (2014) 233506

13. G. Gottstein, Physical foundations of materials science, Springer, Berlin; New York, 2004.

14. J. Friedel, Dislocations, 1st English ed., Pergamon Press, Oxford, New York, 1964.

15. H. Hatano, Journal of Applied Physics 48 (10) (1977) $4397-4399$.

16. S. Y. S. Hsu, K. Ono, H. Hatano, Materials Science and Engineering 38 (2) (1979) $187-191$.

17. C. R. Heiple, S.H. Carpenter, J. Acoustic Emission 6 (3) (1987) $177-237$.

18. M.A. Krishtal, D. L. Merson, M.A. Vyboishchik, Russ Metall Met (6) (1987) 86-88.

19. U. F. Kocks, H. Mecking, Progress in Materials Science 48 (3) (2003) $171-273$.

20. A. Vinogradov, D.L. Merson, V. Patlan, S. Hashimoto, Materials Science and Engineering A 341 (1-2) (2003) $57-73$.

21. A. Vinogradov, M. Nadtochiy, S. Hashimoto, S. Miura, Materials Transactions JIM 36 (4) (1995) 496-503.

22. A. Vinogradov, I. S. Yasnikov, Acta Materialia 70 (2014) $8-18$.

23. L.E. Murr, Interfacial Phenomena in Metals and Alloys, Addison-Wesley, London, UK, 1975.

24. J. A. Venables, J. of Physics and Chemistry of Solids 25 (7) (1964) 685-692.

25. M. Ahlers, Metallurgical Transactions 1 (9) (1970) $2415-2428$.

26. J. Christopher, B. K. Choudhary, Phil. Mag. 94 (26) (2014) $2992-3016$.

27. A. Rohatgi, K.S. Vecchio, Materials Science and Engineering: A 328 (1) (2002) 256-266.

28. H. Parvin, M. Kazeminezhad, Comp. Mater. Science 95 (Suppl. C) (2014) 250-255.

29. S. M. Copley, B. H. Kear, Acta Metallurgica 16 (2) (1968) 227-231. 